\title{
Le Massacre des Amazones : une reconnaissance des femmes de lettres?
}

\author{
Nelly SANCHEZ \\ Université de Limoges \\ san.nelly@yahoo.fr \\ https://orcid.org/oooo-0003-1758-1326
}

\section{Resumen}

Le Massacre des Amazones tuvo, en el momento de su aparición en serie, un gran éxito porque reflejaba la inquietud de la sociedad de la Belle Époque ante la importancia creciente de la literatura femenina. Su autor, Han Ryner, no dejó de denunciar el ridículo de estas mujeres que se desnaturalizaban apropiándose de la característica masculina de la escritura ficción. En efecto, no tuvo piedad por las novelistas. Pero en la expresión de su odio, no se dio cuenta de que su obra satírica participaba, de diferentes maneras, en la promoción y el reconocimiento de la escritura femenina.

Palabras clave: Literatura femenina, Belle Époque, misoginia, tipología, reconocimiento.

\section{Résumé}

Si Le Massacre des Amazones connut, dès sa parution en feuilleton, un grand succès, c'est qu'il traduisait l'inquiétude de la société de la Belle Epoque face à l'importance grandissante de la littérature féminine. Son auteur, Han Ryner, n'eut de cesse de dénoncer le ridicule de ces femmes qui se dénaturaient en s'appropriant cet apanage masculin qu'est l'écriture fictionnelle. Il fut en effet sans pitié pour les romancières. Mais tout à l'expression de sa haine, il ne prit pas conscience que son ouvrage satirique participait, de différentes manières, à la promotion et à la reconnaissance de l'écriture féminine.

Mots-clés : Littérature féminine, Belle Époque, misogynie, typologie, reconnaissance.

\begin{abstract}
At the time of its serial publication, The Massacre of the Amazons was a great success because it reflected the concern of the society of the Belle Epoque in the face of the growing importance of women's literature. Its author, Han Ryner, did not cease to denounce the ridicule of these women who denaturalized them-
\end{abstract}

*Artículo recibido el 16/06/2021, aceptado el 6/11/2021. 
selves by appropriating the masculine privilege that is fictional writing. He was indeed ruthless for the novelists. But while expressing his hatred, he did not realize that his satirical work was involved, in different ways, in the promotion and recognition of feminine writing.

Keywords: Women's literature, Belle Époque, misogyny, typology, recognition.

Le Massacre des Amazones est paradoxalement l'ouvrage le plus connu de Jacques Henri Elie Ner (1861-1938), alias Han Ryner, et le moins cité par ses biographes qui aiment surtout à présenter cet auteur comme un philosophe et journaliste français, anarchiste individualiste, pacifiste. L'histoire de la littérature a pourtant retenu ce titre parce qu'il est la première véritable charge contre la présence massive des femmes sur la scène littéraire ; dès sa parution, d'abord en feuilleton dans La Plume à partir d'octobre 1897, il fit en effet grand bruit. Les critiques d'Han Ryner étaient étayées par une lecture attentive des œuvres vilipendées ; La Fronde (Anonyme, $1898:$ 3) nous apprend d'ailleurs qu'il contactait « pour demander par lettre leurs « œuvres complètes » aux femmes écrivains et les assurer du " profond respect de l'homme et de l'absolue impartialité du critique ».

Nous ne manquerons pas de revenir sur l'originalité de cette entreprise qui, au-delà de l'expression d'une misogynie exacerbée, traduit le malaise profond des intellectuels français face à l'émergence d'une littérature féminine jugée spoliatrice de prérogatives et dangereuse pour l'ordre social établi. Plus ambitieux que Barbey d'Aurevilly dont il s'inspira, Han Ryner mit tout en œuvre pour convaincre de l'inanité de l'écriture féminine, à commencer par s'assurer la complicité du public. Mais à trop vouloir forcer le trait, celui-ci n'a-t-il pas au contraire participé à la reconnaissance des femmes de lettres de la Belle Epoque et assuré, bien malgré lui, la diffusion et la promotion de leur littérature ? Le Massacre des Amazones peut en effet apparaître, sous différents aspects, comme un excellent support publicitaire.

\section{1. « Le Massacre des Amazones », le feuilleton de La Plume}

Le hasard joue rarement un rôle dans les colonnes des journaux. En novembre 1897 débutait la publication en feuilleton bimensuel dans $L a$ Plume, à la rubrique "Biographies, portraits, études et souvenirs littéraires ", du Massacre des Amazones. Celle-ci s'acheva, presqu'un an plus tard, en octobre 1898. Pourquoi cette revue symboliste accepta-t-elle de faire paraître cet ensemble de critiques pamphlétaires contre les femmes écrivains ? Son équipe de rédaction comptait pourtant des littératrices de talent comme Marie Krysinska, promotrice du vers libre et la féministe Manöel de Grandfort... Selon une note conservée dans la compilation complète de ces articles, parue en 1899 chez Chamuel, c'est d'abord avec l'étude intitulée «Bas-Bleus », publiée dans l'hebdomadaire Demain, que débuta Le 
Massacre des Amazones. Elle était consacrée aux derniers romans de Paul Georges, de Jean Laurenty et de Paul Junka, « trois variétés assez distinctes de l'écœurante espèce » (Ryner, 1899: 7). Et celle-ci « souleva des protestations et des encouragements » (Ryner, 1899:6) telles que Han Ryner poursuivit son analyse de l'écriture féminine mais dût changer de journal. Cette critique initiale reparut donc dans La Plume, mais sous un titre nouveau "Première rencontre ». Elle constitua, dans la version finale, le deuxième chapitre du Massacre des Amazones. D’autres articles, toujours consacrés à la production féminine aussi bien poétique que romanesque suivirent dans ce périodique. Si rien n'explique ce changement de revue il est certain que cette conception agressive de la critique littéraire séduisait Léon Deschamps, le directeur de La Plume. Cette revue de jeunes était, comme le rappelle Hélène Millot, "à l'affût de la nouveauté », curieuse de suivre " l'évolution du mouvement littéraire » (Millot, 2002 : 2). En laissant libre cours à sa muflerie, à sa misogynie, Han Ryner ne faisait que traduire l'inquiétude grandissante de ses homologues qui se sentaient menacés dans leurs prérogatives par le nombre grandissant des femmes de lettres dans les colonnes des journaux et dans le catalogue des éditeurs. Le Massacre des Amazones proposait une présentation et un décryptage de ce phénomène en portant au grand jour le nom de ses promotrices.

En presque une année, Han Ryner éreinta ainsi près de deux cents littératrices. Il n'hésita pas, dans un désir d'exhaustivité, à débusquer l'auteure cachée derrière un pseudonyme masculin. Le lecteur attentif connut désormais le véritable sexe de Max Lyan, de Jean Aicard ou de Jean Bertheroy... Afin de mieux servir son attaque, Han Ryner (1899 : 95) ne résista pas au plaisir d'étaler leur vie privée : « En 1863, Mlle Ernestine Drouet publia un recueil intitulé Caritas. Puis elle devint Mme William Mitchel [...]. En 1897, veuve, en retraire, sa fille mariée, elle a donné un second volume, L'Ame Française ». Cela n'inquiéta pas la rédaction de La Plume qui était prête à recevoir les polémiques pourvu qu'elles fissent avancer le débat sur l'avenir de la littérature - plus particulièrement celui du roman en crise après la faillite du naturalisme - qui peinait à se réinventer. Si Han Ryner avait conscience que ses propos au vitriol entraîneraient des réactions, parfois vives, il n'avait pas réalisé qu'il offrait à un grand nombre de ces femmes de lettres une visibilité exceptionnelle et parfois inespérée. Beaucoup n'étaient que d'obscures « ouvrières des Lettres », pour reprendre la terminologie d'Ellen Constans, car elles alimentaient en feuilletons les quotidiens et les revues. Il était même le prétexte idéal pour qu'elles prennent la parole et se fassent ainsi davantage connaître... Parmi les lettres ouvertes que publia La Plume, se trouve celle de Jean Laurenty, pseudonyme de Mlle Fernande Lasmastres, qui priait Léon Deschamps d’insérer ses lignes « pour éviter un peu de bave sur (sa) dignité littéraire » (Laurenty, 1897 : 792). Un 
certain Albert Pradel (1898 : 315) encouragea même les littératrices à se révolter contre « l'incompréhensif $\mathrm{M}$. Ner » en se servant des mêmes armes que celui-ci car elles savaient fort bien manier l'esprit gaulois.

Le premier article que signa Han Ryner parut en novembre 1897 dans La Plume, « La Veillée d'armes », il portait en sous-titre « Études critiques sur les bas-bleus d'aujourd'hui » et était suivi d'une citation extraite du tome $\mathrm{V}$ de Les Euvres et les hommes, «Les Bas-bleus » de Barbey d'Aurevilly (1878) :

La première punition de ces jalouses du génie des hommes a été de perdre le leur... La seconde a été de n'avoir plus le moindre droit aux ménagements respectueux qu'on doit à la femme. Vous entendez, Mesdames ? Quand on a osé se faire amazone, on ne doit pas craindre les massacres sur le Thermodon (Ryner, 1899: 1).

Grâce à cette citation mise en épigraphe, Han Ryner se plaçait sous l'égide de l'auteur des Diaboliques, réputé pour sa viscérale misogynie, et justifiait ainsi la virulente diatribe à laquelle il allait régulièrement se livrer. Mais contrairement à Barbey d'Aurevilly qui consacrait un chapitre à une littératrice et se limitait ainsi à 26 noms, celui-ci entendait, plus ambitieux, s'en prendre à toutes celles qui se mêlaient d'écrire. Dans cet extrait choisi, les femmes de lettres ne sont pas autrement désignées que comme des pêcheresses (la jalousie étant, rappelons-le, un des sept péchés capitaux) et des amazones, deux statuts qui en faisaient les ennemies de la morale et de la civilisation -du patriarcat. Face à cette menace bicéphale, Han Ryner se posait donc en champion de l'ordre et en censeur. Dans cette première critique, à valeur programmatique, il définissait ce qu'était un « bas-bleu » : pédante et prétentieuse, c'est une femme « qui repousse son rôle naturel et, [...] fait l'homme. [...] Ce qui constitue le bas-bleu ou l'amazone, c'est qu'un léger développement de ce qui semble viril en elle lui fait croire qu'intellectuellement elle est un homme » (Ryner, 1899:4-5). Il trouvait également méprisable toutes celles qui faisaient de la littérature un métier et, du livre, une simple marchandise. Cette activité ne devait procéder, à ses yeux, que d'une vocation, d'un talent. Avait-il conscience qu'il s'en prenait directement à Louise d'Alq et son Anthologie féminine (1893) ? Dans sa préface « De l'instruction des femmes et la carrière littéraire », celle-ci expliquait, en effet, que l'écriture était l'une des meilleures manières de gagner sa vie sans déchoir...

Pendant toute une année, Han Ryner s'appliqua donc à fustiger les femmes de lettres en qui il ne voyait «que des petites filles sottes et bavardes, des folles bonnes pour la Salpêtrière, ou des animaux divers » (Millot, $2002: 6$ ). Afin de montrer l'omniprésence du « sexe faible » aussi bien dans la presse (chronique mondaine, feuilleton) que dans la littérature (poésie, roman, théâtre...), il ne manqua jamais ni de les nommer ni de citer leurs 
œuvres. Le lecteur devait prendre conscience de la gravité de la situation : la femme de lettres était partout ! Pour fédérer l'opinion, il choisit d'adopter une démarche taxinomique et entendit établir un classement thématique. Sans doute entendait-il démontrer que la frivolité féminine se retrouvait jusque dans la création romanesque. Il révéla ainsi que les littératrices s'étaient approprié les sujets à la mode : "Anglomanie », "Une pointe en Franco-Russie », " Les Cantinières » désignaient celles qui étaient animées d'une ferveur patriotique, "En Enfance » faisait référence aux auteures mettant en scène des enfants. Grâce à cette typologie, Han Ryner montrait que non seulement la femme de lettres n'était plus un phénomène isolé mais aussi qu'elle s'était parfaitement intégrée au paysage littéraire, qu'elle participait à l'évolution des idées et qu'elle était devenue, par conséquent, un des rouages de la vie intellectuelle. Les homologues de Han Ryner avaientils pris la pleine mesure de ce phénomène ? S’ils reprirent ses idées, ils s'appliquèrent en revanche à gommer toute identité, préférant souligner le profond bouleversement du paysage des lettres que leur existence entraînait. Charles Maurras en parla comme d'une «émeute de femmes » (Décaudin, 1993 : 94), Paul Flat (1909: 1) comme du « groupement serré de celles qui tiennent la plume ». Han Ryner initia également une longue série de recensements des littératrices dont celui de La Revue Bleue, en 1905, et de Je sais tout, en 1907.

Souligner la médiocrité de l'écriture féminine permettait à Han Ryner de rassurer ses confrères : si l'ennemie était omniprésente, elle n'était pas de taille à rivaliser. Dès lors, la dimension ironique du titre de son pamphlet, Le Massacre des Amazones, se fait jour. Ainsi Han Ryner compara ces femmes qu'il se plaisait à infantiliser aux amazones. Il n'eut de cesse de souligner le décalage entre ces figures mythiques et ses contemporaines, jugées ridicules dans leurs efforts pour imiter les hommes. Se souvenait-il sans doute que la satire, en plus de dénoncer, avait fonction de corriger les défauts car comment rappeler leur statut d'éternelle mineure à ces littératrices sinon en adoptant une posture pédagogique ? Pour preuve, l'avis qu'il formula sur Éternel Pierrot de Mlle Berthe Dolor : « Vous avez tort, petite Berthe Dolor, de faire de dilemme le synonyme d'alternative. [...] Je crois inutile, mademoiselle, de continuer la pénible correction de votre devoir » (Ryner, 1899 : 83). Quant à Mme Caro, elle « a lu en bonne élève Alexandre Dumas fils. Elle répète ses leçons d'une voix sans éclat, ânonnante » (Ryner, 1889 : 170). Il ne manqua jamais de se moquer des morales par trop simplistes et récurrentes : le lecteur fut ainsi informé que Mme Gevin-Cassal, « outre le respect de l'argent, [...] enseigne l'amour filiale, le patriotisme, la docilité surtout et que railler est bien vilain »(Ryner, 1899: 130). Il railla bien sûr la naïveté des intrigues comme Le Secret d'Hermine de Mme Astié de Valsayre, où « il y a là, figurez-vous ! un salaud de prince allemand, - marié, s’il vous plaît ! - qui, sous un faux nom, vient nous espionner et promettre le mariage à un ange féminin et français et lui foutre un gosse dans 
le ventre. Acte inouï et bien particulièrement prussien » (Ryner, 1899 : 101102).

Parmi les lieux communs qui fusent exploités par Han Ryner, il convient de signaler celui de la faible constitution féminine qui expliquerait la médiocrité de sa production littéraire. Ce thème semble surtout avoir servi de prétexte pour multiplier les obscénités. Le succès du Massacre des Amazones ne repose d'ailleurs pas tant sur les préjugés qui font fonction d'arguments que sur un style satirique et un registre familier, volontiers graveleux. Les portraits qui sont brossés ne sont pas, sans rappeler par leur férocité, Les Caractères de La Bruyère. Et ce sont de véritables caricatures qui sont données à lire ; Han Ryner n'hésita pas à forcer le trait en assimilant la prolixité d'une romancière à de l'incontinence. Ainsi, dans le chapitre «Quelques mères gigognes », on peut lire que

Le cerveau de Jeanne France [...] est atteint d'une maladie désagréable qu'on situe généralement autre part et dont je croyais la vieillesse féminine exempte. Elle a laissé couler quarante-trois volumes, et le flux continue. J'ai étudié les flueurs recueillies par La Baronne de Langis. Voici le résultat de l'analyse : liquide blanchâtre, tirant parfois sur le jaune, presque insipide et presque inodore (légère fétidité rance). Principaux animalcules en suspension : le vieillard sublime, la jeune fille chaste, la mère séduite qui expie, l'officier séduisant et instruit (Ryner, 1899: 112-113).

Tous les moyens étaient bons pour faire rire et établir ainsi une relation de complicité avec son lectorat qu'il voulait amateur de gauloiseries. Le registre scatologique ne le rebuta pas : «Mme Hector Malot est une adoratrice du veau d'or [...]. Dès que l'idole relève la queue, la prêtresse tend au bon crottin métallique des mains frémissantes et [...] répète l'oraison jaculatoire de l'abbé Alberoni devant le derrière de Vendôme, qui devait être le derrière de la fortune : O culo di angelo ! » (Ryner, 1899 :154-155). Il ne se priva jamais du plaisir d'une grivoiserie :

Et ses sens,

Reconnaissant enfin l'amour qui les embrase,

S'éveillent tout-puissants.

A ces sens tout-puissants, vous reconnaissez immédiatement Hercule et vous songez à celui de ses travaux qui le ferait soigner aujourd'hui comme satyriaque. Vous êtes loin du compte : Mme Jean Bertheroy nous présentait la pudique Psyché (Ryner, 1899: 79).

Bousculant les règles de la bienséance et de la galanterie, Han Ryner s'inscrivait en total décalage avec le ton policé des autres contributions de La Plume. Volontiers satirique et corrosif, il laissait libre cours à sa mauvaise humeur, à son indignation, transformant ainsi ses critiques littéraires en autant d'exutoires. Malgré leur longueur, ces écrits sont fort proches du 
billet d'humeur car leur auteur ne s'interdit rien, pas même la mauvaise foi, pour le plus grand bonheur de son public. A juste titre, Jean Laurenty (1897: 792) se révolta, dans sa lettre ouverte, publiée en 1897, contre « le brillant pamphlétaire » qui, « de sa scrupuleuse exactitude de pion » prenait des fautes typographiques pour « des incorrections de syntaxe ».

\section{Le revers du pamphlet}

Il serait exagéré de penser qu'aucune femme de lettres ne trouva grâce aux yeux de Han Ryner. On peut certes considérer comme épargnées toutes celles qui ne furent jamais mentionnées mais ce serait réducteur car celui-ci formule parfois -très rarement-, d'une phrase lapidaire, un semblant de compliment. Ainsi les œuvres de Marthe Stiévenard, malgré leur " facilité banale », offrent parfois « un sourire (qui lui fut) sympathique ou un geste (qui lui) parut éloquent » (Ryner, 1899 : 255). Une lecture attentive du Massacre des Amazones révèle cependant que les quelques littératrices à s'attirer les bonnes grâces de l'auteur sont les poétesses. Pour ce dernier, Tola Dorian ne mérite le titre de « poète » que pour la dernière pièce des Vespérales, car il dit admirer son " mouvement lyrique et certains détails » (Ryner, 1899: 34). Et si «la poésie de Marie Caussé est trop souvent presque aussi balbutiante que la prose rimée de Francis Jammes », il est « charmé de sa sincérité timide et gracieuse » (Ryner, 1899: 87). Mais gare aux littératrices qui, comme Noël Bazan, sont des « poètes insincères » dont les vers « abondent en souvenirs livresques » (Ryner 1899 : 247)! A trop copier un maître, elles en deviennent ridicules à l'instar de Louise Ducot qui a dédicacé à Sully-Prudhomme ses Rêves d'exil car elle lui doit « beaucoup de qualités extérieures et d'apparences de talent » (Ryner, 1899 : 57). Comment leur en vouloir puisqu'il est admis que « le bas-bleu, singe de l'homme, traduit ordinairement en grimaces les physionomies qu'il voit de près » (Ryner, 1899 : 140) ? Comment s'étonner alors que les « alexandrins grandiloquents et naifs » de Mme Caro-Delvaille aient « quelque chose comme du Hugo inharmonieux et gauche » (Ryner, 1899: 80) ? Mais Han Ryner ne pardonne pas à ces littératrices de passer de la poésie au roman. Il ne manque pas d'épingler Jane de la Vaudère, " poète manqué [qui] s’imagine écrire en prose » (Ryner, 1899 : 15) et qui cherche à copier Marcel Prévost, ou bien Daniel Lesueur, féministe et journaliste à La Fronde, chez qui « le poète est moins méprisable que le romancier » (Ryner, 1899:67-68).

Comment expliquer cette indulgence vis-à-vis des poétesses et cette virulence à l'encontre des romancières ? Ces réactions sont à mettre en regard de la traditionnelle distribution des genres littéraires en fonction des sexes ; en transgressant cette répartition, la femme de lettres ne peut que se dénaturer. Sans qu'il ne le formule jamais, Han Ryner rappelle pourtant que la poésie est l'apanage de la nature féminine, laquelle est alors caractérisée par son irrationnalité, sa sensibilité exacerbée, son manque de maîtrise. Le 
roman est une prérogative masculine. Influencé par la démarche romanesque réaliste et naturaliste ainsi que par la théorie antique des tempéraments -l'homme est un être de raison et de culture-, Han Ryner (1899: 171) estime que « la création refaite par la synthèse est déjà œuvre virile ", aussi la femme de lettres qui écrit un roman se rend coupable de vouloir faire l'homme. Celle-ci pense, en effet, « qu'un léger développement de ce qui semble viril en elle fait croire qu'intellectuellement elle est un homme » (Ryner, 1899:4-5). Toutes celles qui se sont livrées à une réflexion ont sombré dans le ridicule, à commencer par Mme Jousselin qui se plaignit que plusieurs de ses idées avaient été publiées « dans des livres signés Fontenelle » (Ryner, 1899 : 231) ! Pour mieux se moquer de leurs prétentions intellectuelles, Han Ryner leur consacra le chapitre « Je pense, donc je suis ». Qu'elle le veuille ou non, la femme est « plus apte à comprendre la pensée d'autrui, au moins dans son détail infini » (Ryner, 1899 : 171), sa nature la porte à l'empathie, au ressenti, plus qu'à l'observation et à la réflexion. La poésie se prête donc mieux que tout autre genre littéraire, à l'effusion, au lyrisme ; d'ailleurs l'écriture féminine ne devrait pas avoir d'autre finalité que l'expression des sentiments. Comment ne pas rapprocher cette réflexion de la série d'articles intitulée « Le Romantisme féminin » que Charles Maurras publiera dans Minerva en 1903 ? Commentant les vers d'Anna de Noailles, de Renée Vivien, de Lucie Delarue-Mardrus et de Gérard d'Houville, il saluait à chaque fois, " l'influence persistante des romantiques sur un brillant esprit féminin » (Maurras, 1903 : 17). Il est intéressant de constater que le genre poétique, en cette fin de $19^{\mathrm{e}}$ siècle, ne connaissait plus qu'une diffusion confidentielle : les maisons d'édition jadis florissantes périclitaient. L'engouement du public allait désormais au roman. En assignant la prosodie, désormais mineure et confidentielle à la Femme, Han Ryner n'entendait-il pas surtout évincer des concurrentes sur le marché de l'édition?

Quelques romancières s'attirèrent tout de même ses louanges : parmi elles, il faut compter Julia Daudet qui « est le fruit le plus exquis d'une vie à la fois mondaine et intelligente, la réalisation délicieuse d'un idéal connu » (Ryner, 1899 : 40). Sans son chapitre introducteur, il reprit même la définition du bas-bleu formulée par cette littératrice : « Ce que nous appelons le bas-bleu, la femme se servant d'un art comme d'une originalité très voulue, en faisant un moyen d'effet ou de satisfaction vaniteuse » (Ryner, 1899:3). Mais cette admiration et cette déférence étaient loin d'être dénuées d'intérêt car Han Ryner était en train de traduire un ouvrage - Vie d'enfant de Batisto Bonnet - avec son époux, Alphonse Daudet. A la lumière de ces données, toute marque de bienveillance devient dès lors suspecte. Dans l'une de ses premières critiques, "Les Cygnes noirs », Han Ryner rendit compte du talent de Rachilde, de son " éloquence passionnée, abondante », de son « expression [...] souvent évocatrice » car celle-ci « excelle à certains tableaux 
moitié de réalité, moitié de cauchemar » (Ryner, 1899 : 17-18) même s'il estima fort médiocre son roman Les Hors Nature et considérait que comme toutes les femmes de lettres « son génie semble surtout lyrique ; (il voulait dire) puissant et désordonné » (Ryner, 1899:17). Que penser de cet hommage ambigu ? Rachilde faisait partie des co-fondateurs du jeune Mercure de France, promoteur du mouvement symboliste, comme l'était La Plume. Faut-il voir là une manœuvre pour se ménager une entrée future dans les pages de cette revue ? La journaliste Séverine est également épargnée ; elle figure dans les pages consacrées aux « Fille, femme ou veuve » et elle présente « une admirable nature, faite d'humour et de lyrisme. Elle a la fantaisie imprévue et elle vibre [...] à toutes les émotions » (Ryner, 1899 : 160). Il eût été délicat de froisser une consœur qui partageait ses convictions pacifistes et anarchistes. Quelques aristocrates échappèrent à sa muflerie, au nombre desquelles la comtesse Tascher de la Pagerie, la duchesse d'Uzès célèbre pour son amitié avec l'anarchiste Louise Michel -laquelle est également épargnée-, Mme de Rute, petite-nièce de Napoléon ${ }^{\mathrm{er}}$, fondatrice et rédactrice en chef des Matinées Espagnoles... Napoléon III avait même dû expulser de France cette parente qui menaçait, par ses idées trop libérales et son esprit frondeur, son autorité impériale. Nous sommes loin de toute considération littéraire... le pamphlétaire se doublait-il d'un intriguant ? En tout cas, il faisait preuve de peu de courage en s'en prenant plus volontiers à des auteures que l'on peut qualifier de « mineures ».

Mais aussi inique que soit la distinction que Han Ryner établit entre les femmes de lettres qu'il cita, il n'instaura pas moins une hiérarchie qui dépasse la simple typologie thématique. Il organisait suivant une échelle de valeurs - toute personnelle certes -, cette littérature féminine, lui reconnaissait des qualités, des compétences... et surtout des défauts. Par conséquent, ses rares louanges mettaient en lumière certains noms qui apparaissaient dès lors comme autant de modèles à suivre pour qui ne voulait pas être taxé de bas-bleu. C'est ce qu'expliquait le critique et folkloriste Paul de Beaurepaire-Froment dans Le Feu Follet de 1899 : " malgré quelques jugements trop sévères, il ressort de la lecture du livre d'Han Ryner qu'il a rendu justice aux femmes écrivains qui avaient vraiment de la valeur ou qui montraient quelque valeur » (de Beaurepaire-Froment, 1899:380). Une lettre que publia La Plume, lettre intitulée « En descendant de cheval » abonde dans ce sens. Son auteur, "Une Amazone », remerciait Han Ryner d'avoir attiré son attention sur Les Chansons éternelles de Jeanne Marni et de l'avoir " aidée sinon à sentir du moins à définir (la) beauté redoutable et attirante » (Une Amazone, 1898 : 623) de cet ouvrage. Les femmes de lettres citées par Han Ryner, qu'il avait vilipendées, acquéraient soudain une nouvelle importance. En premier lieu, leur présence dans les pages du Massacre des Amazones les distinguait de celles qui en étaient absentes et les sauvaient ainsi de l'oubli ou de l'anonymat. Ensuite, leur nom était autrement 
considéré : ils servaient à illustrer, à éclairer et à préciser les propos de l'auteur. Leur caricature, à cause des défauts qu'elles personnifiaient, faisait figure d'archétype, de modèle original. C'est d'ailleurs ce qu'exprimait, en substance, un contributeur anonyme de La Revue des revues du $1^{\mathrm{er}}$ avril 1899, lorsqu'il rendit compte de la parution du Massacre des Amazones : " Il y en a d'absolument inconnues, si inconnues même qu'elles me paraissent imaginaires. Mais leur humilité ne désarme pas la colère de M. Han Ryner qui châtie si vigoureusement qu'on se demande en vérité s'il n'a pas pris à cœur de restaurer la littérature féminine en France » (La Revue des revues, 1889 : 199). La typologie, ainsi que la hiérarchisation des talents féminins élaborées au fil des pages, amenaient désormais à classer Le Massacre des Amazones parmi les ouvrages de référence, aux côtés des dictionnaire et des anthologies. C'est encore vrai aujourd'hui puisque ce titre est précieux pour les chercheurs, non pas tant pour les infâmies consignées, mais pour le nom des femmes de lettres, leur pseudonyme, le titre des ouvrages qu'il contient. Il est devenu un outil de référence pour qui veut glaner des informations sur une littératrice aujourd'hui tombée dans l'oubli ou seulement étudier l'évolution de la figure de l'amazone littéraire comme dans Femmes et littérature. Une histoire culturelle dirigée par Martine Reid (2020).

La publication en feuilleton du Massacre des Amazones dura jusqu'en octobre 1898, et s'acheva avec "Quelques parasites », où sont évoqués quelques hommes de lettres, dans le onzième chapitre de la version éditée. L'absence de la mention « À suivre » avec laquelle s'achevait chaque livraison, indique que Han Ryner avait volontairement mis un point final à cette critique. Il est intéressant de noter un autre détail, le changement de signature : les articles étaient signés initialement « Henri Ner », ce n'est qu'à partir de juillet 1898 qu'apparaît « Han Ryner » alors qu'il usait de ce pseudonyme depuis 1896. C'est d'ailleurs sous cet allonyme que paraîtra l'ouvrage en 1899. Doit-on conclure que c'est au cours de l'été 1898 que l'auteur décida de publier Le Massacre des Amazones et de le considérer non plus comme une production journalistique mais comme une œuvre littéraire à part entière ? En changeant de patronyme, il copiait ces femmes de lettres si décriées qui publiaient également sous pseudonyme... Avant la mise sous presse, il procéda à quelques modifications et ajouts dont le plus significatif est le long sous-titre qu'il retint : « Etudes critiques sur deux cents bas-bleus contemporains ». En donnant ce chiffre, il établissait le tout premier recensement des femmes de lettres de son époque et, surtout bornait son champ d'analyse, ce qui changeait totalement le caractère de ses critiques littéraires. Il avait conscience de cette nouvelle finalité puisqu'il ajouta cinq chapitres inédits : «Les Frondeuses » consacré aux journalistes et notamment aux contributrices du journal de Marguerite Durand, La Fronde, « plus mal 
renseignée que L'Eclair ou Le Matin » (Han Ryner, 1899 : 186), "Primées », « Je pense, donc je suis », « Au hasard de la massue » et « Paix équitable ». Ce dernier chapitre qui fait figure de conclusion,

Revient aux contradictions qui structurent l'ensemble de son raisonnement : d'un côté, il veut se montrer magnanime et déclare qu'après tout, écrire n'est pas seulement une activité masculine; de l'autre, il reconduit avec aplomb le principe de la différence sexuelle, une différence naturalisée et essentialisée, qui a en réalité sous-tendu ses critiques (Reid, $2017: 307$ ).

Han Ryner devait, en effet, donner une cohérence à cette compilation d'articles en dégageant une synthèse et, par là même, exposer son point de vue sur les femmes de lettres. Mais le fit-il vraiment ? On peut en douter car il acheva son ouvrage par une série de questions qui exprimait surtout son incertitude :

L'affranchissement économique et politique de la femme sera-t-il accompagné de son affranchissement esthétique ? L'esprit féminin se dégagera-t-il de l'imitation de l'esprit viril [...] ? [...] Mais les exceptionnelles qui osent se montrer elles-mêmes deviendront un peu plus nombreuses, et, parmi des œuvres intéressantes, nous donneront peut-être quelques chefs-d'œuvre (Ryner, $1899:$ 263-264).

Rien, à ce jour, n'explique cette décision de réunir en volume Le Massacre des Amazones. Han Ryner offrait au lecteur la possibilité d'apprécier la logique des chapitres jusque-là scindés en plusieurs livraisons. Cette unité se faisait au détriment leur spontanéité de billet d'humeur, ils étaient désormais déconnectés de l'actualité. Les romans, les recueils de poésie analysés au moment de leur parution, étaient datés, dépassés, oubliés pour certains... La dimension synchronique de ces critiques littéraires qui en fit leur succès cédait la place à une dimension diachronique plus intéressante. Si les titres cités - et critiqués - apparaissaient, au moment de leur parution, comme autant de preuves de l'inhabileté de la femme de lettres, ils représentaient désormais autant de phases, de strates dans l'évolution non seulement de leur auteure mais également de l'écriture féminine d'une époque, illustrées par des archétypes, des modèles. Han Ryner ébauchait, sans le vouloir, une histoire de cette littérature. Il mettait en lumière ses thématiques, ses figures majeures et ses modèles. Plutôt que de parler de filiation ou d'influence, Han Ryner s'en tint au préjugé - emprunté à Barbey d'Aurevilly qui veut qu'une « amazone est presque toujours expliquée quand on connaît l'écrivain qu'elle croit admirer et qu'elle méprise assez pour l'imiter » (Ryner, 1899 : 140). Il n'eut, en effet, de cesse de débusquer le modèle qui inspirait le bas-bleu cloué au pilori. Hélène Vacaresco, à l'instar de Tola Dorian, « se laisse prendre au clinquant des Orientales et les lourdeurs barbares de Leconte de Lisle brillent assez pour lui paraitre de l'or » (Ryner, 1899 : 72), 
et « Jean Bertheroy prend-elle fatidique pour un synonyme de fatale ou bien songe-t-elle aux bavardages célèbres des Bijoux indiscrets ? » (Ryner, 1899 : 79). Et « l'œuvre maîtresse de Paul Aigremont s'appelle Monté-Léone. Comme le titre l'indique naïvement, c'est un démarquage de Monte-Cristo. Quelques incidents empruntés aux Mystères de Paris et au Juif-Errant viennent corser l'intrigue trop simple du père Dumas » (Ryner, 1899 : 123). Il consacra même un chapitre entier, " Fille, femme, veuve » à ces littératrices dont Mme Hector Malot, Judith Gautier, Mme Georges Renard, qui, vivant dans l'ombre d'un grand homme, le vampirisaient. Ainsi, Jane Catulle-Mendès qui « sous prétexte de chroniques et de contes, [...] (vendait), depuis son mariage, de nombreuses virgules de Mendès »(Ryner, 1899: 157-158). En voulant dénoncer ces imitations grossières, Han Ryner ne faisait que souligner la culture de ces femmes et leur faculté de réinterpréter et d'interpréter l'objet littéraire. Il prouvait surtout que les femmes pouvaient s'approprier la littérature, les esthétiques, et en étaient des actrices à part entière. La crise que traversa le roman jusqu'à la veille de la Première guerre mondiale valida cet état de fait que niait Han Ryner et bon nombre de ses contemporains. Certains, optimistes comme Jules Bois, estimaient en effet que le roman survivrait

À condition qu'il traduise de plus en plus près la vérité [...] et ceci grâce à l'appoint de la femme moderne [...]. Des sentiments inconnus jusqu'ici sont nés [...] ils nous reposeront du banal adultère ou du libertinage décidément rebattu. [...] Depuis peu tant de femmes extraordinaires se sont manifestées dans le roman et le poème !... Par elles, l'un et l'autre sont sûrs d'une magnifique renaissance (Le Cardonnel, $1903: 76$ ).

Volontiers racoleuse et provocante, la série d'articles qui constitua Le Massacre des Amazones contribua, par les protestations qu'elle provoqua, au succès de son auteur et, par voie de conséquence, de La Plume. Han Ryner amena-t-il, au fil de ses critiques satiriques, le lecteur à méditer sur le danger que représentait l'invasion de la scène littéraire par les femmes de lettres ou ne fit-il que l'amuser tout en confirmant sa prééminence intellectuelle et sociale? Quel que soit son dessein, le soin qu'il mit à recenser les littératrices, à les cataloguer pour établir la première typologie de la production féminine dénote une grande fascination pour celles qui, en se détournant de la poésie pour le roman, transgressaient la frontière des genres. D'autre part, l'absence de véritable conclusion à la fin du Massacre des Amazones laisse supposer qu'il n'eut pas de véritable avis tranché sur ce qui, à ses yeux, représentait une menace réelle pour la Société. Mais lui-même ne transgressa-t-il pas les conventions sociales en se présentant comme anarchiste, pacifiste et anticlérical dans une France revancharde et désignée comme la fille aînée de l'Eglise ? Quelles que soient ses réelles intentions, il mettait en lumière le profond malaise d'une société qui, bien qu'éprise de modernité, demeurait farouchement attachée à la séparation des sexes. 
Cette confusion des rôles sociaux qu'engendrait la littérature féminine était en effet perçue comme un signe de décadence et de la crise de masculinité, comme l'expliqua Anne-Lie Maugue (2001) dans L'Identité masculine en crise au tournant du siècle.

\section{RÉFÉRENCES BIBLIOGRAPHIQUES}

ANONYME, (1898) : «Les Revues ». La Fronde, 29 avril, 3.

ALQ, Louise d' (1893) : Anthologie féminine : anthologie des femmes écrivains, poètes et prosateurs depuis l'origine de la langue française jusqu'à nos jours. Paris, Bureaux des Causeries familières.

Amazone, Une (1898) : « En descendant de cheval », in La Plume, 229, 623.

BEAUREPAIRE-Froment, Xavier de (1899) : «Bibliographie », in Le Feu Follet, décembre-janvier, 380 .

BESSIERE, Lesly (2008) : «Les romancières françaises, 1870-1900 : approche à travers l'exemple de Jeanne Marni », in Genre \& Histoire, 2. URL: http://journals.openedition.org/genrehistoire/266

DECAUDIN, Michel (1994) : «Bacchantes ou Amazones ? Romancières de 1900 ». Cahiers de l'Association internationale des études françaises, 46, 93-104.

FLAT, Paul (1909) : Nos Femmes de lettres. Paris, Perrin \& Cie (coll. Librairie académique).

LAURENTY, Jean (1897) : «À propos du Massacre des Amazones ». La Plume, 792.

LE CARDONNEL, Georges \& Charles VELLAY (1905) : La Littérature contemporaine. Paris, Mercure de France.

LLOZE, Evelyne (2007) : « Éléments de réflexion sur la question du genre dans la poésie moderne et contemporaine », in Delphine Naudier et Brigitte Rollet (dir.), Genre et légitimité culturelle. Quelle reconnaissance pour les femmes ? Paris, L'Harmattan (coll. « Bibliothèque du féminisme »), 74-97.

MAUGUE, Anne-Lise (2001 [1987]) : L'Identité masculine en crise au tournant du siècle. Paris, Payot (coll. «Poche »).

MAURRAS, Charles (1903) : « Romantisme féminin ». Minerva, $1^{\mathrm{er}}$ mai, 16-26.

Millot, Hélène (2002) : « La réception critique de la poésie féminine dans les petites revues littéraires du tournant du siècle : L'exemple de La Plume (18891905) », in Masculin / Féminin dans la poésie et les poétiques du XIX ${ }^{e}$ siècle. DOI : https://doi.org/10.4000/books.pul.6471

Planté, Christine (2015) : La Petite sour de Balzac. Essai sur la femme auteur. Lyon, PUL.

PlantÉ, Christine (2003) : «La place des femmes dans l'histoire littéraire : annexe, Ou point de départ d'une relecture critique ? ». Revue d'Histoire littéraire de la France, 3, juillet-septembre, 655-668.

PrADEL, Albert (1898) : «Si les Amazones voulaient ! », La Plume, 218, 315. 
REID, Martine (2017) : «Réflexions sur Le Massacre des Amazones d'Han Ryner », in Andrea del Lungo et Brigitte Louichon (dir.), La littérature en bas-bleus III. Romancières en France de 1870 à 1914. Paris, Garnier, 305-314.

RYNER, Han (1899) : Le Massacre des Amazones, Paris, Chamuel Éditeur.

SANCHEZ, Nelly (2010) : Images de l'Homme dans les romans de Rachilde et de Colette (1884-1943). Sarrebruck, Éditions Universitaires Européennes.

VON KUlESSA, Rotraud (2007) : "La Femme auteur à l'époque 1900 : débats et tentatives de légitimation », in Delphine Naudier et Brigitte Rollet (dir.), Genre et légitimité culturelle. Quelle reconnaissance pour les femmes ? Paris, L'Harmattan, 99-119. 